\title{
Non-typhoidal Salmonella isolates from livestock and food samples, Kolkata, India
}

\author{
Sudhanthiramani Sudhanthirakodi ${ }^{\star \star}$, Priyanka Jain ${ }^{2 \star}$, Utpal Kumar Chattopadhyay ${ }^{1}$, Shanta Dutta ${ }^{2}$ \\ ${ }^{*}$ Both authors have contributed equally. \\ ${ }^{1}$ Department of Microbiology, All India Institute of Hygiene and Public Health, 27 \& 27B, JC Block, Salt Lake City, Sector 3, \\ Kolkata- 700098. West Bengal, India; \\ ${ }^{2}$ Division of Bacteriology, National Institute of Cholera and Enteric Diseases, P-33 CIT Road, Scheme XM, Beliaghata, \\ Kolkata-700010, West Bengal, India.
}

\begin{abstract}
Objective: Non-typhoidal Salmonella (NTS) is a major foodborne pathogen in both developed and developing countries. The study was undertaken to determine the prevalence, serotype distribution and antimicrobial resistance profiles of NTS isolates from livestock and food samples collected in and around Kolkata during 2012-2013.

Materials and methods: A total of 500 samples (animal, poultry and raw food) from 12 different places randomly collected in and around Kolkata were processed as per standard procedures and analyzed for NTS. Serotyping of the isolates was done followed by determination of antimicrobial resistance profiles and Minimum inhibitory concentrations following disc diffusion and E-test methods.

Results: A total of 53 (10.6\%) NTS isolates were recovered, with highest isolation from chicken (40\%), followed by beef and poultry cloacal samples (22\% each). Total 11 Salmonella serovars were identified with predominance of Salmonella enterica serovar Typhimurium (S. Typhimurium) (30.2\%) followed by S. Kentucky (22.6\%), S. Idikan (15.1\%) and other serovars. This is the first report of isolation of $S$. Idikan from India. Thirty-two (60.4\%) isolates were pan susceptible to all drugs tested. Percentage distribution of resistant NTS against antimicrobials were: nalidixic acid (24.5\%), ampicillin (17\%), amoxicillin (17\%), streptomycin (15.1\%), tetracycline (11.3\%), chloramphenicol (9.4\%), co-trimoxazole (9.4\%) and ciprofloxacin (5.7\%). Only one isolate (S. Senftenberg) was resistant to both fluroquinolones and third generation cephalosporins. Multidrug resistance ( $\geq 3$ classes of antimicrobials) was observed in $13.2 \%$ NTS isolates.
\end{abstract}

Conclusion: High prevalence of NTS in poultry and meat samples in Kolkata poses a potential risk of foodborne disease in humans. J Microbiol Infect Dis 2016;6(3): 113-120

Key words: Non-typhoidal Salmonella, antimicrobial resistance, animals, poultry, food samples

\section{INTRODUCTION}

Non-typhoidal Salmonella (NTS) are one of the most important zoonotic bacterial foodborne pathogens of humans that cause diarrhoea, bacteraemia and focal suppurative infections [1]. According to the Centers for Disease Control (CDC, Atlanta), approximately 1.2 million illnesses due to NTS, with 23,000 hospitalizations and 450 deaths are reported annually in the United States alone [2]. However, the actual disease occurrence is often underestimated in many developing countries like India due to lack of an effective surveillance system. Among more than 2500 serovars (serotypes) of Salmonella enterica, some serovars like Salmonella enterica serovar Typhimurium (S. Typhimurium) and $S$. Enteritidis have a broad host range, while some are associated with specific animal reservoirs such as $S$. Gallinarum in poultry, $S$. Abortus-ovis in sheep, $S$. Dublin in cattle and $S$. Choleraesuis in pigs. These animal-adapted serotypes show a much higher predilection for causing invasive disease in humans [1]. Contaminated raw foods (meat, eggs, milk) and infected animals are important sources of human infections [1,3-5]. In general, antimicrobial therapy is not recommended for gastroenteritis caused by NTS in humans, however, it is indicated for invasive salmonellosis $[1,2]$. Therapeutic and non-therapeutic use of various antibiotics in food-producing animals has led to the emergence of antimicrobial resistance (AMR) among NTS, which is a global concern and has restricted the availability of antimicrobials for human use [1,2]. Data on the prevalence of NTS in livestock animals and their products provides epidemiologi-

Correspondence: Shanta Dutta, Scientist G, Division of Bacteriology, National Institute of Cholera and Enteric Diseases, P-33 CIT Road, Scheme XM, Beliaghata, Kolkata 700010, India. Email: shanta1232001@yahoo.co.in

Received: 08.09.2015, Accepted: 08.04.2016

Copyright (C) Journal of Microbiology and Infectious Diseases 2016, All rights reserved 
cal insight and help to predict the probable disease burden of that region due to the zoonotic nature of the organism. Knowledge on the current trend of antimicrobial resistance profiles of the NTS would help in formulating appropriate treatment (empirical) policies, which would prevent emergence and transmission of the resistant strains.

Several studies on the prevalence and AMR profiles of NTS from animals and foods of animal origin have been documented from all over the world including India [4-8]. However, there is scarcity of data from the eastern part of India. Only two reports were available from Kolkata which described isolation and antibiotic susceptibility pattern of NTS from poultry specimens (carcass, meat and organs) and the other study documented the molecular characterization of NTS isolates from chicken viscera, meat and egg shell collected from local shops $[9,10]$. With this background information, the present study was undertaken with the aim to determine the prevalence, serotype distribution and AMR profiles of NTS isolates from livestock and food samples collected from various (around 12) places in and around Kolkata.

\section{MATERIALS AND METHODS}

\section{Ethics statement}

The present study was reviewed and approved by the Animal Ethical Committee of National Institute of Cholera and Enteric Diseases (NICED), Kolkata.

\section{Sample size and period}

This is a cross-sectional descriptive study. A total of 200 livestock samples comprising of poultry cloacal swabs $(n=50)$, goat rectal swabs $(n=50)$ and cattle rectal swabs $(n=100)$ as well as 300 raw food samples comprising of chicken $(n=50)$, mutton $(n=50)$, beef $(n=50)$, pork $(n=50)$, unpasteurized poultry eggs $(n=50)$ and unpasteurized milk $(n=50)$ collected randomly over eight month period from July 2012 to February 2013 were included in this study for further analysis (Table 1).

\section{Study area}

The poultry cloacal swabs and rectal swabs from animals (goat and cattle) were sampled from livestock reared in 16 private local farms, located 50$100 \mathrm{~km}$ away from the main city of Kolkata. The poultry samples were collected from 2 farms each in Singur, Bhadreswar and Habra; the cattle and goat rectal samples from 2 farms each in Singur, Bhadreswar, Habra, Bally and Dankuni (Figure 1,
A-E). The total number of livestock around Kolkata was not known. Selection of the farms and livestock was done on the basis of the free choice of the owner who cooperated during our study. Two different farms were visited on each of 8 months. Random sampling was done. Five to ten samples per category were collected from each of the 10 animal farms and 6 poultry farms. Ten rectal swabs from cattle were collected from each of the 10 different animal farms visited (10×10=100 samples). Ten goat rectal swabs were collected from each of 4 farms $(10 \times 4=40)$ and 5 samples from each of 2 other farms $(5 \times 2=10)$. Six animal farms were common from where cattle and goat rectal swabs were obtained. Similarly, 10 poultry cloacal swabs were collected from each of 4 poultry farms $(10 \times 4=40)$ and 5 samples from each of two other poultry farms $(5 \times 2=10)$. Each animal/poultry was sampled only once. The rectal swabs from pigs could not be collected in this study due to the lack of cooperation from piggery farm owners.

The food samples including raw meat, whole eggs and whole milk were randomly selected and purchased from 10 retail shops, 14 butcher shops and 6 supermarkets located at 7 different places within the city of Kolkata which included Beliaghata, Belgachia, Shyambazar, Sealdah, Colootola street, Burrabazar and NewMarket (Figure 1, F-L). Each of the 30 shops was sampled once during the study period and approximately 10 samples were collected from each shop. The farms from which the animals were brought to the retail/ butcher shops and the supermarkets for sale was not known.

\section{Sample collection and transport}

All livestock samples (rectal and cloacal swabs) were collected under the proper guidance and supervision of the farm owners. Sterile cotton swab was inserted by a veterinarian into the animal rectum using sterile gloves and rotated twice along the wall of the intestine without causing any harm to the animals. In case of cloacal samples, both the wings of the birds were held with one hand so that the tail portion remained in upper direction. Sterile cotton swab was then inserted into the cloacae and rolled inside the cloacae several times. Both the rectal and cloacal swabs were transported in Cary-Blair transport medium (Difco, Maryland, USA) at room temperature to the NICED laboratory for further investigations.

In case of food samples, around $25-30 \mathrm{~g}$ of raw meat, $20-30 \mathrm{ml}$ unpasteurized milk and whole eggs were collected aseptically twice a week, packed 
carefully in sterile polyethylene bags or falcon tubes, labeled properly with date, place of collection, origin etc and transported to the NICED laboratory immediately placing inside a thermo flask containing ice cubes.

\section{Sample processing for isolation of NTS}

All the samples were processed according to standard protocol: Isolation of Salmonella spp. from food and animal feaces [11]. Briefly, $25 \mathrm{~g}$ of the meat samples were homogenized with $225 \mathrm{ml}$ of buffered peptone water (BPW) (Difco Maryland, USA) for pre-enrichment in the ratio of 1 in 10 and incubated at $37^{\circ} \mathrm{C}$ for $18-24$ hours. After incubation, $1 \mathrm{ml}$ of pre-enrichment broth culture was inoculated aseptically into Selenite Cysteine broth (SC) (Difco, Maryland, USA) and $100 \mu$ into Rappaport Vassili- adis Soy peptone broth (RVS) (Merck, Germany) for selective enrichment. Further the inoculated SC and RVS broths were incubated at $37^{\circ} \mathrm{C}$ for 18 hours and at $41.5^{\circ} \mathrm{C}$ for 24 hours respectively. The overnight enriched samples were inoculated onto MacConkey Agar (MAC), Xylose Lysine Deoxycholate Agar (XLD) and Hektoen Enteric Agar (HEA) (Difco Maryland, USA) for colony selection.

In case of egg and milk samples, one ml each of milk and egg sample (edible portion of egg after breaking open the shell aseptically) were transferred into $9 \mathrm{ml}$ of BPW in the ratio of 1 in 10 . The rectal/ cloacal swabs were directly inoculated into BPW followed by incubation at $37^{\circ} \mathrm{C}$ for 24 hours. Rest of the procedures was similar to those mentioned earlier.

Table 1. Distribution of non-typhoidal Salmonella (NTS) isolates $(n=53)$ among study samples collected in and around Kolkata during July 2012 to Feb 2013.

\begin{tabular}{|c|c|c|c|c|c|c|}
\hline \multirow{2}{*}{$\begin{array}{l}\text { Sl. } \\
\text { No. }\end{array}$} & \multirow{2}{*}{$\begin{array}{l}\text { Types of the study } \\
\text { samples }\end{array}$} & \multirow{2}{*}{$\begin{array}{c}\text { Samples } \\
\text { processed } \\
(\mathrm{n})\end{array}$} & \multirow{2}{*}{$\begin{array}{c}\text { Positive } \\
\text { samples } \\
\text { n (\%) }\end{array}$} & \multicolumn{3}{|c|}{ Distribution of NTS as per Kauffmann-White Scheme } \\
\hline & & & & $\begin{array}{l}\text { NTS serovars } \\
(\mathrm{n})\end{array}$ & $\begin{array}{c}\text { NTS } \\
\text { serogroup }\end{array}$ & $\begin{array}{l}\text { Antigenic formulae of } \\
\text { the serovars }\end{array}$ \\
\hline A. & Livestock samples & 200 & $16(8)$ & & & \\
\hline \multirow[t]{3}{*}{1.} & \multirow[t]{3}{*}{ Poultry (cloacal swab) } & \multirow[t]{3}{*}{50} & \multirow[t]{3}{*}{$11(22)$} & Idikan (8) & G & 1,13,23: i: 1,5 \\
\hline & & & & Kentucky (2) & $\mathrm{C}_{2}-\mathrm{C}_{3}$ & $8, \underline{20}: \mathrm{i}: \mathrm{z}_{6}$ \\
\hline & & & & Typhimurium (1) & B & $1,4,[5], 12: \mathrm{i}: 1,2$ \\
\hline \multirow[t]{4}{*}{2.} & \multirow[t]{4}{*}{ Cattle (rectal swab) } & \multirow[t]{4}{*}{100} & \multirow[t]{4}{*}{$5(5)$} & Teko (2) & $\mathrm{H}$ & {$[1], 6,14,[25]: d: e, n, z_{15}$} \\
\hline & & & & Enteritidis (1) & $\mathrm{D}_{1}$ & 1,9,12: g,m: - \\
\hline & & & & Typhimurium (1) & $4 ; B$ & $1,4,[5], 12: \mathrm{i}: 1,2$ \\
\hline & & & & Weltevreden (1) & $\mathrm{E}_{1}$ & $3,\{10\}\{\underline{15}\}: r: z_{6}$ \\
\hline 3. & Goat (rectal swab) & 50 & $0(0)$ & no isolation (0) & - & \\
\hline B. & Food samples & 300 & $37(12.3)$ & & & \\
\hline \multirow[t]{6}{*}{1.} & \multirow[t]{6}{*}{ Chicken } & \multirow[t]{6}{*}{50} & \multirow[t]{6}{*}{$20(40)$} & Typhimurium (12) & B & $1,4,[5], 12: \mathrm{i}: 1,2$ \\
\hline & & & & Bareilly (4) & $\mathrm{C}_{1}$ & $6,7, \underline{14}: y: 1,5$ \\
\hline & & & & Cerro (1) & $\mathrm{K}$ & $\underline{6}, \underline{14}, 18: z_{4}, z_{23}:[1,5]$ \\
\hline & & & & Infantis (1) & $\mathrm{C}_{1}$ & $6,7, \underline{14}: \mathrm{r}: 1,5$ \\
\hline & & & & Kentucky (1) & $\mathrm{C}_{2}-\mathrm{C}_{3}$ & $8, \underline{20}: \mathrm{i}: \mathrm{z}_{6}$ \\
\hline & & & & Virchow (1) & $\mathrm{C}_{1}$ & $6,7,14: r: 1,2$ \\
\hline \multirow[t]{2}{*}{2.} & \multirow[t]{2}{*}{ Beef } & \multirow[t]{2}{*}{50} & \multirow[t]{2}{*}{$11(22)$} & Kentucky (9) & $\mathrm{C}_{2}-\mathrm{C}_{3}$ & $8, \underline{20}: \mathrm{i}: \mathrm{z}_{6}$ \\
\hline & & & & Salmonella spp. (2) & $\mathrm{F}$ & 11: -:- (untypable) \\
\hline \multirow[t]{3}{*}{3.} & \multirow[t]{3}{*}{ Mutton } & \multirow[t]{3}{*}{50} & \multirow[t]{3}{*}{$5(10)$} & Bareilly (2) & $\mathrm{C}_{1}$ & $6,7,14: y: 1,5$ \\
\hline & & & & Typhimurium (2) & $\mathrm{B}$ & $1,4,[5], 12: \mathrm{i}: 1,2$ \\
\hline & & & & Senftenberg (1) & $\mathrm{E}_{4}$ & 1,3,19: g,[s],t:- \\
\hline 4. & Pork & 50 & $0(0)$ & no isolation (0) & - & \\
\hline 5. & Milk & 50 & $1(2)$ & Enteritidis (1) & $D_{1}$ & 1,9,12: g,m: - \\
\hline \multirow[t]{2}{*}{6.} & Poultry egg & 50 & $0(0)$ & no isolation (0) & - & \\
\hline & Total & 500 & $53(10.6)$ & & & \\
\hline
\end{tabular}




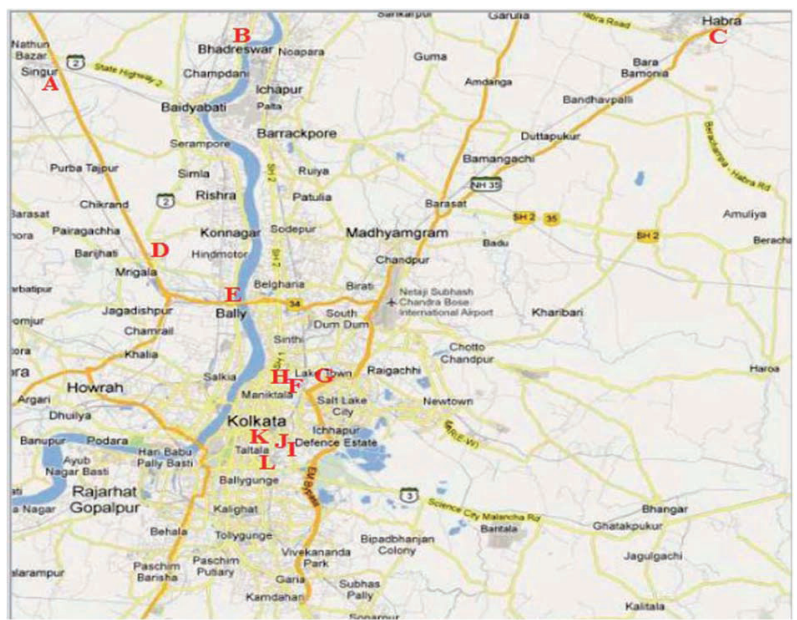

A: Singur; B: Bhadreswar; C: Habra; D: Dankuni; E: Bally F: Beliaghata; G: Belgachia; H: Shyambazar; I: Sealdah J: Colootola street; K: Burrabazar; L: NewMarket

Figure 1. Study sites in and around Kolkata from where samples have been collected during July 2012 to Feb 2013.

A-E, Farms from where livestock were sampled;

F-L, Raw food samples collection centers.

\section{Isolation and identification of NTS}

The presumptive identification of NTS was based on colony characters i.e., non-lactose fermenting colonies on MAC, red colonies with black centres on XLD agar and green colonies with black centres on HEA \& set of standard biochemical tests which included triple sugar iron, lysine iron agar, mannitol fermentation, motility, indole, citrate and urease tests.[12] Confirmation was done by serotyping the isolates using Salmonella poly- and monovalent $\mathrm{O}$ and $\mathrm{H}$ antisera (Denka Seiken Pvt. Ltd, Tokyo, Japan). The Salmonella serotype was determined following White- Kauffmann-Le Minor scheme [13].

\section{Antimicrobial susceptibility test and determination of Minimum Inhibitory Concentrations (MIC)}

The isolates were tested for their antimicrobial susceptibility using following 17 antimicrobial discs (BD, Maryland, USA); ampicillin, chloramphenicol, tetracycline, co-trimoxazole, nalidixic acid, ciprofloxacin, norfloxacin, ofloxacin, amikacin, gentamicin, streptomycin, ceftazidime, cefotaxime, ceftriaxone, aztreonam, azithromycin and amoxyclav, on MuellerHinton agar by Kirby-Bauer disk diffusion method. MICs of drugs to which the isolates were resistant, were determined by E-test (AB biodisk, Solna, Sweden) following manufacturer's instructions. Results were interpreted according to Clinical and Laborato- ry Standards Institute (CLSI) guidelines. Escherichia coli ATCC 25922 was used as control strain [14].

\section{RESULTS}

\section{Prevalence of NTS in livestock and food samples}

Out of 500 samples processed, 53 samples were found to be contaminated with NTS (Table 1). In livestock, NTS prevalence was $8 \%$ (16 out of 200) and it was more frequently isolated from poultry cloacal $(22 \%)$ samples than cattle $(5 \%)$ and goat $(0 \%)$ rectal swab samples. The total prevalence of NTS in food samples was $12.3 \%$ (37 out of 300 ) with highest contamination found in chicken $(40 \%)$, followed by beef $(22 \%)$, mutton $(10 \%)$ and milk $(2 \%)$. No Salmonella were isolated from pork and poultry eggs.

\section{Distribution of NTS serovars}

Among the 53 NTS isolated, 11 different serovars were identified. Distribution of serovars showed that chicken samples harboured 6 different serovars, followed by 4 serovars in cattle rectal swab, 3 serovars each in mutton and poultry clocal swabs samples and 1 serovar each in milk and beef samples. Two isolates from beef samples were untypable (Table 1). The predominant serovar among all samples was $S$. Typhimurium $(n=16,30.2 \%)$ followed by $S$. Kentucky $(n=12,22.6 \%), S$. Idikan $(n=8,15.1 \%)$ and $S$. Bareilly $(n=6,11.3 \%)$. Seventy-five percent of $S$. Typhimurium and $S$. Kentucky were recovered from chicken and beef samples respectively. S. Idikan isolates were recovered only from poultry cloacal samples and was the predominant serovar in this category. Rare serovar like S. Teko was isolated from cattle rectal samples.

\section{Antimicrobial resistance profiles of NTS serovars}

The percentage of AMR in NTS isolates and the MICs of antimicrobials are shown in Table 2. The most common antimicrobial to which the different serovars of Salmonella were resistant was nalidixic acid $(n=13,24.5 \%)$; followed by ampicillin $(n=9$, $17 \%)$, amoxyclav $(n=9,17 \%)$, streptomycin $(n=8$, $15.1 \%)$, tetracycline $(n=6,11.3 \%)$, chloramphenicol $(n=5,9.4 \%)$ and co-trimoxazole $(n=5,9.4 \%)$. Lower resistance were observed for ciprofloxacin, norfloxacin, ofloxacin and gentamicin $(n=3,5.7 \%)$. Only one isolate ( $S$. Senftenberg) was observed to be resistant to third generation cephalosporins (ceftazidime, cefotaxime, ceftriaxone) and aztreonam. All isolates were susceptible to amikacin and azithromycin. Overall $60.4 \%(n=32)$ of the isolates 
were pan susceptible to all antimicrobials tested, while $39.6 \%(n=21)$ isolates showed resistance to at least one antimicrobial. Altogether seven resistance profiles were found among the NTS isolates (Table 3 ). Multidrug resistance (resistance to 3 or more classes of antimicrobials) was observed among 7 $(13.2 \%)$ isolates comprising 3 different serovars $S$. Typhimurium $(n=4), S$. Kentucky $(n=2)$ and $S$. Senftenberg $(n=1)$ (Table 3).

Table 2. Antimicrobial resistance and the minimum inhibitory concentrations (MICs) of antimicrobials among NTS isolates from the study samples, Kolkata during July 2012 to Feb 2013.

\begin{tabular}{|c|c|c|c|c|c|c|c|c|c|c|c|c|c|c|}
\hline \multirow[t]{3}{*}{$\begin{array}{l}\text { Antimicrobials }{ }^{a} \\
\text { (Disk potency in } \mu \mathrm{g})\end{array}$} & \multirow[t]{3}{*}{$\begin{array}{l}\text { MIC range } \\
\text { in } \mu \mathrm{g} / \mathrm{mL}\end{array}$} & \multicolumn{12}{|c|}{$\begin{array}{l}\text { Distribution of various NTS serovars among AMR } \\
\text { Isolates }(n=53)\end{array}$} & \multirow[t]{3}{*}{$\begin{array}{l}\text { Distribution of AMR in } \\
\text { NTS Isolates } n(\%)\end{array}$} \\
\hline & & 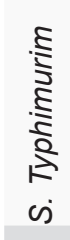 & 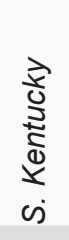 & $\frac{\sqrt{\frac{\pi}{2}}}{\text { c) }}$ & 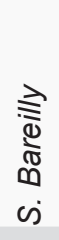 & 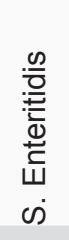 & $\begin{array}{l}\frac{0}{\oplus} \\
\text { c) }\end{array}$ & 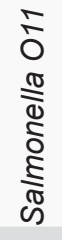 & $\begin{array}{l}0 \\
\frac{0}{d} \\
0 \\
\text { ci }\end{array}$ & $\underset{\dot{\infty}}{\stackrel{\infty}{\stackrel{\infty}{N}}}$ & 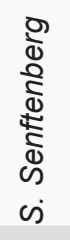 & $\begin{array}{l}\text { oे } \\
\frac{\delta}{0} \\
\dot{J} \\
\text { ci }\end{array}$ & 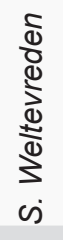 & \\
\hline & & (16) & (12) & (8) & (6) & $(2)$ & (2) & (2) & (1) & (1) & (1) & (1) & (1) & \\
\hline $\mathrm{Na}(30)$ & $>256$ & 7 & 2 & 0 & 0 & 1 & 0 & 0 & 1 & 1 & 1 & 0 & 0 & $13(24.5)$ \\
\hline$A(10)$ & $>256$ & 0 & 0 & 8 & 0 & 0 & 0 & 0 & 0 & 0 & 1 & 0 & 0 & $9(17.0)$ \\
\hline Amc (30) & $>256$ & 0 & 0 & 8 & 0 & 0 & 0 & 0 & 0 & 0 & 1 & 0 & 0 & $9(17.0)$ \\
\hline$S(10)$ & 64 & 4 & 2 & 0 & 0 & 0 & 0 & 0 & 1 & 0 & 1 & 0 & 0 & $8(15.1)$ \\
\hline $\mathrm{T}(30)$ & $32->256$ & 4 & 2 & 0 & 0 & 0 & 0 & 0 & 0 & 0 & 0 & 0 & 0 & $6(11.3)$ \\
\hline$C(30)$ & $32->256$ & 4 & 0 & 0 & 0 & 0 & 0 & 0 & 0 & 0 & 1 & 0 & 0 & $5(9.4)$ \\
\hline Q (25) & $>32$ & 4 & 0 & 0 & 0 & 0 & 0 & 0 & 0 & 1 & 0 & 0 & 0 & $5(9.4)$ \\
\hline Gm (10) & 16 & 0 & 2 & 0 & 0 & 0 & 0 & 0 & 1 & 0 & 0 & 0 & 0 & $3(5.7)$ \\
\hline Cip (5) & $>32$ & 0 & 2 & 0 & 0 & 0 & 0 & 0 & 0 & 0 & 1 & 0 & 0 & $3(5.7)$ \\
\hline Nor (10) & $24->256$ & 0 & 2 & 0 & 0 & 0 & 0 & 0 & 0 & 0 & 1 & 0 & 0 & $3(5.7)$ \\
\hline Ofx (5) & $>32$ & 0 & 2 & 0 & 0 & 0 & 0 & 0 & 0 & 0 & 1 & 0 & 0 & $3(5.7)$ \\
\hline Ctx (30) & $>256$ & 0 & 0 & 0 & 0 & 0 & 0 & 0 & 0 & 0 & 1 & 0 & 0 & $1(1.9)$ \\
\hline Caz (30) & 16 & 0 & 0 & 0 & 0 & 0 & 0 & 0 & 0 & 0 & 1 & 0 & 0 & $1(1.9)$ \\
\hline Cro (30) & $>256$ & 0 & 0 & 0 & 0 & 0 & 0 & 0 & 0 & 0 & 1 & 0 & 0 & $1(1.9)$ \\
\hline Atm (30) & 96 & 0 & 0 & 0 & 0 & 0 & 0 & 0 & 0 & 0 & 1 & 0 & 0 & $1(1.9)$ \\
\hline An (30) & $2-8$ & 0 & 0 & 0 & 0 & 0 & 0 & 0 & 0 & 0 & 0 & 0 & 0 & $0(0.0)$ \\
\hline Azm (15) & $2-8$ & 0 & 0 & 0 & 0 & 0 & 0 & 0 & 0 & 0 & 0 & 0 & 0 & $0(0.0)$ \\
\hline
\end{tabular}

${ }^{a}$ A: ampicillin; Amc: amoxyclav; An: Amikacin; Atm: aztreonam; Azm: azithromycin; C: chloramphenicol; Caz: ceftazidime; Cip: ciprofloxacin; Cro: ceftriaxone; Ctx: cefotaxime; Gm: gentamicin; Na: nalidixic acid; Nor: norfloxacin; Ofx: ofloxacin; Q: co-trimoxazole; S: streptomycin; T: tetracycline. 
Table 3. Antimicrobial resistance profiles of NTS isolates from study samples, Kolkata

\begin{tabular}{|c|c|c|c|c|}
\hline SI. No. & Antimicrobial resistance profile ${ }^{a}$ & Salmonella serotypes & Source & Number of isolates \\
\hline 1. & ACSNaCipNorOfxCazCtxCroAtmAmc & S. Senftenberg ${ }^{b}$ & Mutton & 1 \\
\hline 2. & TNaCipNorOfxGmS & S. Kentucky ${ }^{\mathrm{b}}$ & Poultry cloacal swab & 2 \\
\hline \multirow[t]{2}{*}{3.} & CTQSNa & S. Typhimurium ${ }^{b}$ & Poultry cloacal swab & 1 \\
\hline & & & Chicken & 3 \\
\hline 4. & $\mathrm{GmSNa}$ & S. Cerro & Chicken & 1 \\
\hline 5. & AAmc & S. Idikan & Poultry cloacal swab & 8 \\
\hline 6. & QNa & S. Infantis & Chicken & 1 \\
\hline \multirow[t]{2}{*}{7.} & $\mathrm{Na}$ & S. Typhimurium & Chicken & 3 \\
\hline & & S. Enteritidis & Milk & 1 \\
\hline
\end{tabular}

aA: ampicillin; Amc: amoxyclav; Atm: aztreonam; C: chloramphenicol; Caz: ceftazidime; Cip: ciprofloxacin; Cro: ceftriaxone; Ctx: cefotaxime; Gm: gentamicin; Na: nalidixic acid; Nor: norfloxacin; Ofx: ofloxacin; Q: co-trimoxazole; S: streptomycin; T: tetracycline.

${ }^{\mathrm{b}}$ Multidrug resistant (resistance to 3 or more antimicrobial classes) isolates

\section{Discussion}

The prevalence of NTS in livestock and foods vary greatly from region to region, even within the same country, which is dependent upon the climatic conditions, hygiene and management practices of farm, handling, processing and storage of raw food. It may also be affected by differences in sampling season, sample type, sampling methods and isolation techniques employed.

In the present study, the percentage of NTS prevalence in poultry cloacal samples was $22 \%$ which was higher than $14.7 \%$ isolation as reported in previous study from India [7]. Studies from Taiwan reported $10.9 \%$ NTS isolation from the same samples [15]. The isolation of NTS in cattle rectal samples $(5 \%)$ in this study was lower compared to the earlier studies from India $(9.7 \%)$ and Addis Ababa $(71.4 \%)[7,16]$.

The total NTS contamination in food samples as observed in this study $(12.3 \%)$ was higher than the report from China $(9.7 \%)$, but was lower than the contamination rates reported from Romania $(22.9 \%)$ and the US $(20.5 \%)[5,6,17]$. Contamination with NTS in $40 \%$ of retail chicken samples in this study was higher than the earlier reports from India, conducted in different cities like Wardha and Patna, reporting isolation of $28.3 \%$ and $23.7 \%$ NTS respectively $[8,18]$. Contamination levels were found variable as reported by numerous studies worldwide; $7.4 \%$ from China, $67.8 \%$ from Romania and $35 \%$ from the US $[5,6,17]$. Salmonella prevalence in beef samples $(22 \%)$ was higher in this study than that re- ported from China $(16.8 \%)$, the US $(6.0 \%)$ and the western part of India $(10.6 \%)[5,17,19]$. For mutton samples, the rate of contamination $(10 \%)$ in this study was lower than reports from earlier studies in China (11.0\%) and India (38.3\%) [5,8]. Likewise, earlier studies from Addis Ababa and India reported higher rate of NTS isolation from milk samples, being $28.6 \%$ and $7.7 \%$ respectively $[16,18]$. No Salmonella was isolated from either pork or poultry eggs in this study, however, reports of NTS isolation from these products have been frequently documented earlier $[3,5,6,17]$.

The distribution of Salmonella serovars identified in this study was heterogenous. Eleven different serovars were identified among the 53 isolates, and two isolates from beef samples remained untypable (Table 1). To the best of our knowledge, $S$. Idikan isolates has not been earlier reported from India from any samples, although it was previously reported from poultry litter and food in Chad, Africa [20]. Isolation of $S$. Teko, another rare serovar was reported earlier only from India, in association with betel leaves [21]. Worldwide Salmonella serovars frequently isolated from poultry include $S$. Enteritidis, S. Infantis and S. Kentucky, $[4,22,23]$ while common serovars isolated from cattle include $S$. Typhimurium, $S$. Montevideo and $S$. Dublin $[4,16,22]$.

In this study, $S$. Typhimurium $(n=12)$ and $S$. Kentucky ( $n=9)$ were the predominant serovars isolated from chicken and beef retail meat category respectively. Similar results were reported by the National Antimicrobial Resistance Monitoring Sys- 
tem, USA [22]. Other common serovars isolated from meat samples from other countries include $S$. Enteritidis, S. Derby, S. Infantis, S. Agona $[5,6,17]$. Since the number of isolates in each serovar was very small, association between sources and the Salmonella serovars could not be established in this study.

Antimicrobial resistance in NTS is a global concern. In the current study, $60.4 \%$ of the isolates showed pan susceptibility. This was in contrast to the reports from China, Romania and the US where, $93.5 \%, 91.9 \%$ and $84.0 \%$, isolates, respectively, showed resistance to at least one antimicrobial. $[5,6,17]$. Resistance to nalidixic acid $(24.5 \%)$ was predominantly observed among the NTS serovars in this study. This level observed was lower than those reported by Mihaiu et al, in Romania $(65.1 \%)$ but higher than earlier reports by Murugkar et al, from North-east India (17.9\%) [6,7]. Resistance to ciprofloxacin and third generation cephalosporins (cefotaxime, ceftazidime and ceftriaxone) was found in 3 $(5.7 \%)$ strains and in $1(1.9 \%)$ strain respectively, in the current study. Higher resistance rates of $25.8 \%$ and $42.95 \%$ for ciprofloxacin were reported from China and Romania, respectively [5,6]. Variable percentage resistance for third generation cephalosporins was reported from China (1.6\%), Romania $(11.4 \%)$ and the US (16\%) $[5,6,17]$. This emerging resistance to fluoroquinolones and third generation cephalosporins is alarming since these are the current drugs of choice for treating invasive salmonellosis in humans [24-27].

Multidrug resistance was found to be $13.2 \%$ $(n=7 / 53)$ in the present study. Higher values of $33.9 \%, 83.2 \%$ and $53.0 \%$, were recorded from China, Romania and the US, respectively $[5,6,17]$. Resistant NTS organisms were mostly isolated from poultry cloacal and chicken meat samples (Table 3). Poultry is found to be the most important reservoir of NTS worldwide and have been implicated in many foodborne outbreaks in humans [1]. Resistance was more commonly observed in S. Idikan $(n=8), S$. Typhimurium $(n=7)$ and $S$. Kentucky $(n=2)$ serovars (Table 3 ). These serovars (e.g.S. Typhimurium, S. Kentucky) are more likely to become multidrug resistant than other serovars (e.g. S. Enteritidis) $[4,22,23]$. In this study, four isolates of $S$. Typhimurium, although were multidrug resistant (MDR; resistant to chloramphenicol, tetracycline, co-trimoxazole, streptomycin and nalidixic acid) but were susceptible to ampicillin. This is in contrast to the typical multidrug resistance profile of $S$. Typhimurium which shows resistance to ampicillin in addition to other drug resistance $[4,5,17]$. It was interesting to note that $S$. Kentucky $(n=2)$ isolates from poultry cloacal swabs were MDR, but the isolates from the beef samples were pan susceptible. MDR S. Senftenberg isolated from goat meat showed resistance to both fluoroquinolones (MIC of ciprofloxacin: $>32 \mu \mathrm{g} / \mathrm{mL}$; ofloxacin: $>32 \mu \mathrm{g} /$ $\mathrm{mL}$; norfloxacin: $>256 \mu \mathrm{g} / \mathrm{mL}$ ) and third generation cephalosporins (MIC of cefotaxime: $>256 \mu \mathrm{g} / \mathrm{mL}$; ceftriaxone: $>256 \mu \mathrm{g} / \mathrm{mL}$ and ceftazidime: $16 \mu \mathrm{g} /$ $\mathrm{mL}$ ) (Table 2). NTS serovars like S. Agona, S. Choleraesuis, $S$. Enteritidis and $S$. Typhimurium showing resistance to both these groups of drugs is on a rise and have been reported from other countries including India [24-27].

In conclusion, the study provides a brief insight into the prevalence, common serovars and AMR profiles of NTS isolates from livestock and food samples in and around Kolkata. High rate of isolation of NTS from poultry, chicken meat and beef poses a potential risk of transmission of these organisms in humans. It was interesting to note that although MDR was more common in NTS isolates globally, it was less prevalent in Kolkata. Nevertheless, isolation of $S$. Senftenberg resistant to both fluroquinolones and third generation cephalosporins give an early warning signals for conducting effective surveillance and judicial use of antimicrobials in food animals for containment of the resistant bacteria.

\section{Acknowledgements}

\section{Conflict of Interest: None to declare.}

Financial Support: The study was supported by the Indian Council of Medical Research (ICMR), New-Delhi intramural fund. ICMR senior research fellowship to $P$. Jain is gratefully acknowledged.

This study was carried out as a dissertation submitted by Dr. S. Sudhanthirakodi for partial fulfillment of MVPH degree. We would also like to thank the animal farm and retail shop owners for their cooperation and assistance in sample collection.

Declaration of Conflicting Interests: The authors declare that they have no conflict of interest.

Financial Disclosure: The study was supported by the Indian Council of Medical Research (ICMR), NewDelhi intramural fund as given above. 


\section{REFERENCES}

1. Hohmann EL. Nontyphoidal Salmonellosis. Clin Infect Dis 2001; 32: 263-269.

2. Centers of Disease Control and Prevention (CDC). National Antimicrobial Resistance Monitoring System-Enteric Bacteria (NARMS): 2011 Human Isolates Final Report. Atlanta, GA: U.S. Department of Health and Human Services, 2013.

3. Suresh T, Hatha AA, Sreenivasan D, Sangeetha N, Lashmanaperumalsamy P. Prevalence and antimicrobial resistance of Salmonella Enteritidis and other Salmonellas in the eggs and egg-storing trays from retail markets of Coimbatore, South India. Food Microbiol 2006; 23: 294-299.

4. Esaki H, Morioka A, Ishihara $\mathrm{K}$, et al. Antimicrobial susceptibility of Salmonella isolated from cattle, swine and poultry (2001-2002): report from the Japanese Veterinary Antimicrobial Resistance Monitoring Program. J Antimicrob Chemother 2004; 53: 266-270.

5. Yu T, Jiang X, Zhou Q, Wu J, Wu Z. Antimicrobial resistance, class 1 integrons, and horizontal transfer in Salmonella isolated from retail food in Henan, China. $\mathrm{J}$ Infect Dev Ctries 2014; 8: 705-711.

6. Mihaiu L, Lapusan A, Tanasuica R, et al. First study of Salmonella in meat in Romania. J Infect Dev Ctries 2014; 8: 50-58.

7. Murugkar HV, Rahman H, Kumar A, Bhattacharyya D. Isolation, phage typing and antibiogram of Salmonella from man \& animals in northeastern India. Indian J Med Res 2005; 122: $237-242$.

8. Moon AH. Studies on: Prevalence and antibiogram of Salmonella species of polluted meat origin. Asiatic J Biotech Res 2011; 2: 447-453.

9. Selvaraj R, Das R, Ganguly S, Ganguli M, Dhanalakshmi S, Mukhopadhayay SK. Characterization and antibiogram of Salmonella spp. from poultry specimens. J Microbiol Antimicrob 2010; 2: 123-126.

10. Selvaraj R, Das R, Ganguly S, Mukhopadhayay SK. Molecular characterization of Salmonella spp. isolated and identified from chicken samples. Int J Pharm Res Bio-Sci 2014; 3: 507-517.

11. World Health Organization (WHO). Global Foodborne Infections Network. Laboratory Protocol: "Isolation of Salmonella spp. from food and animal feaces" (5th Edition), 2010.

(http://www.antimicrobialresistance.dk/data/images/protocols/ isolation_of_salm_220610.pdf)

12. World Health Organization (WHO). Global Foodborne Infections Network. Laboratory Protocol: "Biochemical Identification of Salmonella and Shigella using an abbreviated panel of tests" (3rd Edition), 2010.

(http://www.antimicrobialresistance.dk/data/images/protocols/ gfn_biochem_final.pdf)

13. Guibourdenche M, Roggentin P, Mikoleit M, et al. Supplement 2003-2007 (No. 47) to the White-Kauffmann-Le Minor scheme. Res Microbiol 2010; 161: 26-29.

14. Clinical and Laboratory Standards Institute (CLSI). Performance Standards for Antimicrobial Susceptibility Test- ing; Twenty-Fourth Informational Supplement, M100-S24. Wayne, PA, 2014.

15. Chiu LH, Chiu $\mathrm{CH}$, Horn YM, et al. Characterization of 13 multi-drug resistant Salmonella serovars from different broiler chickens associated with those of human isolates. BMC Microbiol 2010; 10: 86.

16. Addis Z, Kebede N, Worku Z, Gezahegn H, Yirsaw A, Kassa T. Prevalence and antimicrobial resistance of Salmonella isolated from lactating cows and in contact humans in dairy farms of Addis Ababa: a cross sectional study. BMC Infect Dis 2011; 11: 222.

17. White DG, Zhao $S$, Sudler R, et al. The isolation of antibioticresistant Salmonella from retail ground meats. N Engl J Med 2001; 345: 1147-1154.

18. Kaushik P, Anjay, Kumari S, Bharti S K, Dayal S. Isolation and prevalence of Salmonella from chicken meat and cattle milk collected from local markets of Patna, India. Vet World 2014; 7: 62-65.

19. Kshirsagar DP, Singh S, Brahmbhatt MN, Nayak JB. Isolation and molecular characterization of virulence-associated genes of Salmonella from buffalo meat samples in western region of India. IsrJ Vet Med 2014; 69: 228-233.

20. Tabo DA, Diguimbaye CD, Granier SA, et al. Prevalence and antimicrobial resistance of non-typhoidal Salmonella serotypes isolated from laying hens and broiler chicken farms in N'Djamena, Chad. Vet Microbiol 2013; 166: 293-298.

21. Singh BR, Singh $M$, Singh $P$, Babu N, Chandra M, Agarwal RK. Prevalence of multidrug-resistant Salmonella on readyto-eat betel leaves (Paan) and in water used for soaking betel leaves in North Indian cities. J Food Prot 2006; 69: 288-292.

22. Food and Drug Administration (FDA). National Antimicrobial Resistance Monitoring System - Enteric Bacteria (NARMS): 2011 Executive Report. Rockville, MD: U.S. Department of Health and Human Services, 2013. (http://www.fda.gov/ downloads/ AnimalVeterinary/ SafetyHealth/ AntimicrobialResistance/ NationalAntimicrobialResistanceMonitoringSystem/ UCM407962.pdf)

23. Barua H, Biswas PK, Talukder KA, Olsen KE, Christensen JP. Poultry as a possible source of non-typhoidal Salmonella enterica serovars in humans in Bangladesh. Vet Microbiol 2014; 168: 372-380.

24. Menezes GA, Khan MA, Harish BN, et al. Molecular characterization of antimicrobial resistance in non-typhoidal Salmonellae associated with systemic manifestations from India. J Med Micobiol 2010; 59:1477-1483.

25. Su LH, Teng WS, Chen CL, et al. Increasing ceftriaxone resistance in salmonellae, Taiwan. Emerg Infect Dis 2011; 17 1086-1090.

26. Cheung TK, Chu YW, Chu MY, Ma CH, Yung RW, Kam KM. Plasmid-mediated resistance to ciprofloxacin and cefotaxime in clinical isolates of Salmonella enterica serotype Enteritidis in Hong Kong. J Antimicrob Chemother 2005; 56:586-589.

27. Wong MH, Yan M, Chan EW, Biao K, Chen S. Emergence of clinical Salmonella enterica serovar Typhimurium isolates with concurrent resistance to ciprofloxacin, ceftriaxone, and azithromycin. Antimicrob Agents Chemother 2014; 58: 37523756. 